\title{
A Pilot Study Evaluating the Effect of Daily Education by a Pharmacist on Medication Related HCAHPS Scores and Medication Reconciliation Satisfaction
}

\author{
Megan Huebner*, Mary E Temple-Cooper, Melissa Lagzdins, Jun-Yen Yeh \\ Hillcrest Hospital, Cleveland Clinic, USA
}

\begin{abstract}
Purpose: The purpose of this study is to determine if daily pharmacist counseling improves Hospital Consumer Assessment of Healthcare Providers and Systems (HCAHPS) medication scores in a 25 bed medical surgical unit. Secondary objectives included determination of Full-time equivalent (FTE) hours required to complete the task of a pharmacist completing daily counseling and medication reconciliation for each patient on a 25 bed hospital unit, as well as determining if medication reconciliation performed on each patient improved satisfaction survey scores among staff.

Methods: This was a single center, controlled, parallel study in two medical surgical units. Patients included were those admitted to the control or intervention unit, and the primary investigator (PI) completed daily counseling in the intervention unit and counseling once during admission on the control unit. Medication reconciliation was also completed by the $\mathrm{PI}$ on the intervention unit, and satisfaction was assessed through a survey provided to caregivers before and after the study. An FTE analysis was completed to determine the FTE and cost burden to implement this practice model.

Results: A total of 128 patients were included in the study over 27 days. Overall medication communication scores increased by $11.4 \%$ and decreased by $0.9 \%$ in the intervention and the control unit, respectively. Communication about side effects increased by $43 \%(p=0.007)$ and $13.3 \%(p=0.013)$ in the intervention and control units, respectively. A number of medication reconciliation satisfaction endpoints trended towards significance including decreased number of medication misadventures $(p=0.107)$, increased efficiency of patient admission $(p=0.157)$ and decreased interference with patient discharge $(p=0.157)$, and decreased total time to complete the discharge process $(p=0.058)$. The FTE cost analysis indicated that on average, an additional 16 minutes of counseling is required per 3 day admission. Therefore, an additional four to seven FTEs will be required to incorporate this model into our institution.

Conclusion: Daily counseling by a pharmacist resulted in a statistically significant increase in communication about side effect HCAHPS survey scores and an overall increase in medication communication compared counseling once during admission.
\end{abstract}

\section{Background}

Pharmacists provide pharmaceutical care with other healthcare workers to improve medical outcomes for patients. Pharmacists have a responsibility to take an active role in patient care and ensuring patients understand their drug regimens. Medication non-adherence costs the United States of America $\$ 100$ billion and noncompliance has caused 2 million hospital admissions per year [1]. Pharmacists can increase adherence and decrease readmission rates by counseling patients about the purpose, appropriate use, most common side effects and management of side effects for their medications [1]. Pharmacists also improve pharmaceutical care by preventing medication errors [2].

The Center for Medicare and Medicaid (CMS) established reimbursement criteria based on a number of endpoints, including pharmaceutical care and medication communication. Medication communication criteria are measured through a Hospital Consumer Assessment of Healthcare Providers and Systems (HCAHPS) Survey. Institutions seeking CMS reimbursement are required to distribute these surveys to their patients discharged from their hospital.2 The HCAHPS Survey measures medication communication and assesses patient satisfaction with caregiver communication about their medications and potential side effects [2]. Survey scores are based on the percentage of patients that answer "always" to the survey questions. These percentages are then ranked into percentiles. CMS utilizes the HCAHPS survey to standardize measuring patients' perspectives on hospital care. This is a method that allows collection and public reporting of patients' perspectives of care information that will allow comparisons to be made across all hospitals [3].
Thorough and complete admission medication histories are also critical in preventing medication errors. Medication reconciliation (MR) is a tool to aid in admission and discharge of patients. MR ensures all appropriate medications are being discontinued or held upon admission and appropriately restarted at discharge. Medication history discrepancies may increase medication misadventures, increase patient harm and costs and reduce caregiver time with patients while discrepancies are resolved [4]. An accurate and efficient MR process is required to assure success in the process, prevent patient harm and to prevent delays in admission and discharge. Assessment of healthcare team satisfaction with the MR process is essential to assure the best practice is utilized in admitting and discharging patients [2].

Increasing the frequency of medication counseling to patients by pharmacists may contribute to improved HCAHPS scores [5].

*Corresponding author: Megan Huebner, PGY2 Ambulatory Care Resident Cleveland Clinic Health Systems, USA, Tel.: 412-802-8805; E-mail: HUEBNEM@ccf.org

Received July 03, 2013; Accepted August 16, 2013; Published August 22, 2013

Citation: Huebner M, Temple-Cooper ME, Lagzdins M, Yeh J (2013) A Pilot Study Evaluating the Effect of Daily Education by a Pharmacist on Medication Related HCAHPS Scores and Medication Reconciliation Satisfaction. J Biosafety Health Educ 1: 105. doi:10.4172/2332-0893.1000105

Copyright: (c) 2013 Huebner M, et al. This is an open-access article distributed under the terms of the Creative Commons Attribution License, which permits unrestricted use, distribution, and reproduction in any medium, provided the original author and source are credited. 
Citation: Huebner M, Temple-Cooper ME, Lagzdins M, Yeh J (2013) A Pilot Study Evaluating the Effect of Daily Education by a Pharmacist on Medication Related HCAHPS Scores and Medication Reconciliation Satisfaction. J Biosafety Health Educ 1: 105. doi:10.4172/2332-0893.10 00105

Page 2 of 6

Pharmacists may require additional time to counsel patients when increasing the frequency of patient counseling. The feasibility of increased counseling time added to other pharmacists' duties requires an assessment before such a practice is incorporated into daily work flow. Currently, there is no data available to describe the feasibility and time requirement for a pharmacist to complete daily counseling and MR on an adult medical surgical unit. Our primary hypothesis was that pharmacists can be utilized to improve HCAHPS medication communication scores by counseling patients daily versus once during admission. Our secondary hypothesis was that a MR intervention completed by pharmacists would improve healthcare worker satisfaction. This study was conducted at Hillcrest Hospital, a 500-bed Cleveland Clinic community hospital. Researchers conducted the study in two similar, 25-bed, medical surgical units. The Cleveland Clinic Foundation Institutional Review Board reviewed the study protocol and approval was received prior to study initiation.

\section{Methods}

\section{Study Design}

This was a 27 day prospective, pilot-study performed on two similar medical/surgical units within a 500-bed community hospital. The study was composed of three main parts as shown in Figure 1, a patient counseling intervention, MR intervention and satisfaction survey, and a full-time equivalent analysis. The primary objectives of this study were to determine if the initiation of a daily pharmacist-based counseling service improved HCAHPS medication scores compared to HCAHPS medication scores in a second group of patients counseled only once during admission. Secondary objectives included assessment of the number of full time equivalent hours required to complete admission medication counseling, daily and discharge counseling, and MR for each patient on a 25 bed hospital unit. Other secondary outcomes were analyzed, including the number of orders a pharmacist was able to process while doing MR and patient counseling versus how many orders were verified by the pharmacist staff verifying the control unit orders. Finally, we sought to determine if MR performed on each patient in the intervention unit improved satisfaction survey scores among nursing, physician and ancillary staff.

\section{Pharmacy Counseling Intervention}

Patients were included for the pharmacy counseling intervention if they were admitted to either the control or intervention unit and did not have dementia unless a family member was present to receive medication counseling from the pharmacist. Patients were excluded if they were scheduled to be discharged to a skilled nursing facility, rehabilitation facility, long-term acute care facility, or nursing home. The pharmacy counseling intervention was performed on two similar

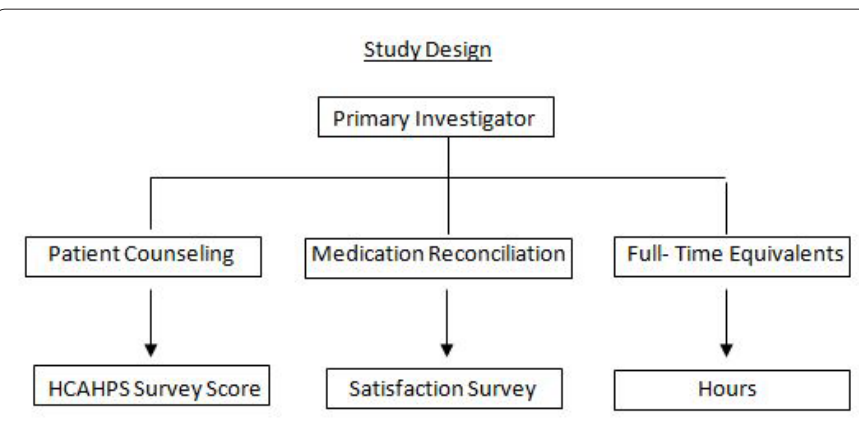

Figure 1: Study Design. medical/surgical units. The control unit was a 25 bed unit where the Primary Investigator (PI) counseled each patient once upon admission. The intervention unit was a 25 bed unit where the PI counseled each patient on a daily basis and performed MR for each patient on admission and at discharge. Patient counseling was completed on these two units by one primary evaluator (pharmacist) for 27 consecutive days from the hours of 10 am to $6 \mathrm{pm}$. Each patient on the control unit was counseled about their medications within 24-48 hours after admission. Medication counseling included the indication for each medication and potential side effects. If the patient was incapable of interpreting or understanding the counseling as assessed by the diagnosis of dementia, a family member received the counseling. Patients on the intervention unit received daily counseling. Counseling occurred within 24 hours of admission, on each day of hospital stay, and prior to discharge. Each day the pharmacist addressed any ongoing medication issues with the patient in addition to providing counseling on new medications. The pharmacist also reviewed discharge medications ordered for the patient and any changes made to the patients' drug regimen as compared to prior to admission. An inpatient note was recorded after each counseling session in the patient's chart documenting the patient counseling for that specific intervention. Patient counseling was not performed by any other pharmacist on either the control unit or intervention unit during the 28 day study to control for bias and variability.

The HCAHP survey was distributed at random to a group of individuals on both the control and intervention units. The Cleveland Clinic hires an independent group to disperse their HCAHP surveys to patients after they are discharged from the hospital. The researchers were blinded regarding which patients received the survey. Selected patients received a phone call after discharge asking them to evaluate their stay at Hillcrest Hospital. The patients could have taken the survey or refused at will. The researchers did not know how many declined the survey, just how many took the survey. The portion of HCAHP survey of interest in this study were the two medication related questions (Appendix 1 and 2). The HCAHP percentage for each question only included the "always" answers or the top-box choice. The percentages of patients who answered "always" to the medication question were given to an independent reporter and were reported to the researchers. The total percentage of participants who answered "always" to one or both of the medication questions of the HCAHP survey were recorded and reported by the quality office and this data was used to determine the difference in HCAHP scores before and after the interventions.

Prescription order verification was completed by the PI for the intervention unit. Stat or immediate orders were required to be verified within fifteen minutes of order entry by the ordering provider. If stat orders were not verified by the PI within fifteen minutes, another pharmacist in the hospital verified the order. Non-stat orders remained in the order verification screen for thirty minutes before a pharmacist outside the intervention unit verified the order [6]. A report was run identifying how many orders were verified by the PI and how many were verified by the other pharmacists.

\section{Medication Reconciliation Intervention and Satisfaction Survey}

MR was performed by a pharmacist for each patient in the intervention unit. MR was performed for each patient in one of two ways. The first method occurred in the emergency room (ER). The ER pharmacist spoke with the patient, patient representatives, the patient's pharmacy or primary physician office to clarify current medications. The emergency room pharmacist completed the MR when they were 
Citation: Huebner M, Temple-Cooper ME, Lagzdins M, Yeh J (2013) A Pilot Study Evaluating the Effect of Daily Education by a Pharmacist on Medication Related HCAHPS Scores and Medication Reconciliation Satisfaction. J Biosafety Health Educ 1: 105. doi:10.4172/2332-0893.10 00105

Page 3 of 6

available. The emergency room pharmacist works seven days on and seven days off. The PI completed MR for all other patients admitted to the intervention unit. A note was added to each patient's chart indicating the MR had been completed. A MR template was used by both the PI and the pharmacist in the emergency room.

MR satisfaction was assessed before and after the MR intervention on the intervention unit. A ten question survey was designed using a likert scale and approved by the IRB as a quality tool. The survey was distributed as part of a quality initiative and was phase one of this study. The survey was distributed to nurses, physicians, and social workers during this phase. Phase two of the MR study involved a reassessment of nurses, physicians, and social workers satisfaction with the MR process after the PI finished the 27 day pilot program. The survey in phase 1 was used to assess the overall evaluation of the current MR process in the hospital during the time prior to the study initiation. The phase 2 survey was distributed within one week of completion of the pharmacist intervention month to reevaluate the overall satisfaction of the caregivers for the MR process in the hospital. The same ten questions were distributed to the same individuals and the differences in answers pre and post intervention were analyzed.

\section{Full-time Equivalent Analysis}

A full-time equivalent analysis was performed in order to assess the feasibility of incorporating the tasks of daily pharmacist counseling and MR into a pharmacy practice model. FTEs were calculated based on the time allotted for the task of the primary evaluator in addition to order entry. The minutes spent on each task were recorded after each patient counseling session (admission, interim, and discharge), each record of the patient note, and each MR during admission and at discharge for the intervention unit. The researchers also recorded the minutes spent counseling on the control unit. Average minutes spent counseling was compared between the control and intervention unit. A cost analysis was completed to determine the financial requirement of implementing a pharmacy practice model similar the one demonstrated in this study. The following assumptions were made to complete our cost-analysis: 500-bed hospital, average of 47 non-ICU adult admissions per day, average length of stay three days.

\section{Statistical Analysis}

The sample size calculation was based on the primary endpoint; change in HCAHPS survey scores before and after the intervention. This calculation was based on $95 \%$ confidence interval, a power set at $80 \%$, and an effect size of $5 \%$ which required a sample size of 64 patients preintervention and 64 patients post-intervention. The 64 pre-intervention patients included the patients completing the HCAHPS survey 1 month prior to the study month. The 64 patients post-intervention included the patients completing the HCAHPS survey during the study. The primary endpoint was the change in percentage of daily counseled patients rating the medication counseling portion of the HCAHP survey as "always" from baseline versus change in percentage from baseline in a second group of patients counseled only on admission. This was analyzed using a Fisher's exact test. The Wilcoxon signed rank matched pairs test was used to analysis the change in HCAHP survey scores within each group compared to their historical HCAHP survey data.

The secondary endpoints included FTE hour calculation required by a pharmacist performing daily counseling in a 25 bed unit versus counseling only on admission on the control unit, MR for each new admission on a 25 bed unit, and the number of orders entered by the PI on the unit during a 10 hour shift. The difference in full-time equivalent requirements was analyzed using a t-test. We hypothesized that an additional four hours or 0.5 FTEs might be required to counsel each patient daily and perform MR on admission and discharge. Also, the change in MR satisfaction in assigned unit from baseline was assessed. The MR satisfaction survey data was analyzed by determining the mode for each question as well as evaluating the change in the percentage of answers for each individual question after the MR intervention. This change was analyzed using the Wilcoxon signed ranks matched pairs test. All data was analyzed using SigmaPlot 10.0 [7]

\section{Results}

One-hundred and twenty-eight patients were included in the study population with 71 on the intervention unit and 58 on the control unit (Table 1). The average age of the patients was 61 years and $62 \%$ were female. The primary admitting diagnoses on the intervention unit were orthopedically related, including patients who were post-hip and knee arthroplasty. Orthopedic admission accounted for $24 \%$ of the admissions included from this unit. The primary admitting diagnoses on the control unit were gastrointestinal related, accounting for $47 \%$ of the patients included from this unit. Approximately 80 patients were excluded secondary to skilled nursing, long-term care, or acute rehabilitation placement at discharge. An additional 10 patients were excluded for communication barriers such as language and hearing.

\section{HCAHPS Counseling}

Two days into the study, the time allotted per day for patient counseling and MR was changed from 10 hours per day (10am to $8 \mathrm{pm})$ to 8 hours per day (10am to $6 \mathrm{pm}$ ). This change was based on the determination the completion of study tasks was feasible in an 8 hour period. A total of 10 and 14 surveys were completed on the intervention and control units, respectively, by patients discharged during the study month (February 2013). The overall change in medication communication scores, as compared to one month prior to the study intervention (January 2013), increased by $11.4 \%$ and decreased by $0.9 \%$ in the intervention and control units, respectively (Figure 2 and 3). The percentage of patients answering always to question 1 (Communication about what the medication is for) decreased on both units compared to January 2013: $26 \%(\mathrm{p}=0.186)$ and $15.2 \%(\mathrm{p}=0.179)$ percent decrease in the intervention and control units, respectively. A statistically significant increase in the percentage of patients who answered "always"

\begin{tabular}{|l|c|c|c|}
\hline Demographic & Intervention Unit & Control Unit & Total \\
\hline Average Age (years) & $63(20-93)$ & $59(25-88)$ & 61 \\
\hline Males & $36(51 \%)$ & $14(24 \%)$ & $50(39 \%)$ \\
\hline Females & $35(49 \%)$ & $44(76 \%)$ & $79(61 \%)$ \\
\hline Total Patients & 71 & 58 & 129 \\
\hline
\end{tabular}

Table 1: Baseline Characteristics.

Percentage of Patients Answering "Always"

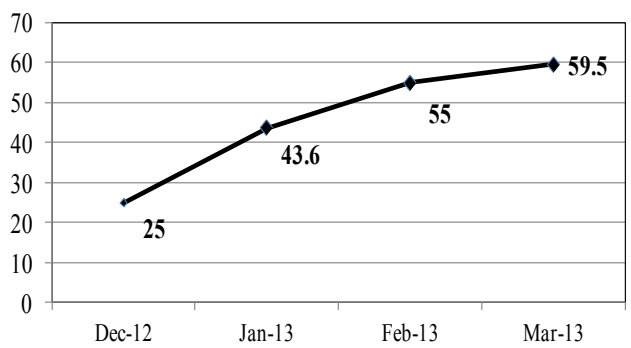

Figure 2: Change in overall medication communication scores from month to month Intervention Unit. 
Citation: Huebner M, Temple-Cooper ME, Lagzdins M, Yeh J (2013) A Pilot Study Evaluating the Effect of Daily Education by a Pharmacist on Medication Related HCAHPS Scores and Medication Reconciliation Satisfaction. J Biosafety Health Educ 1: 105. doi:10.4172/2332-0893.10 00105

Page 4 of 6

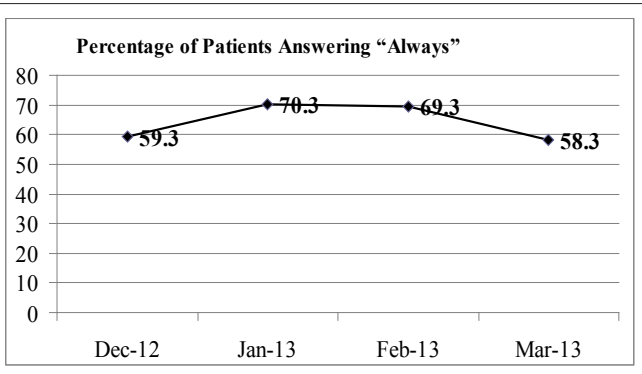

Figure 3: Change in overall medication communication scores from month to month in Control Unit.

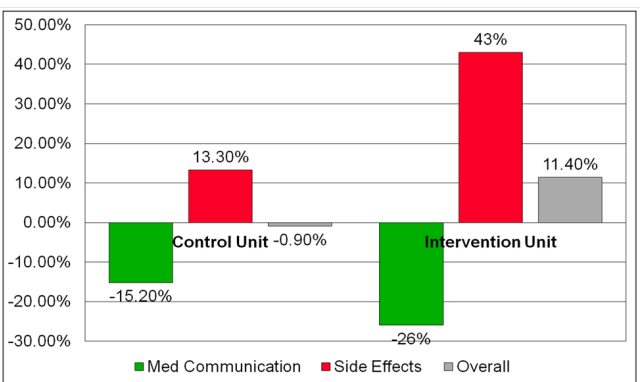

Figure 4: Total percentage change from January to February 2013 on Intervention vs. Control.

to question 2 (Communication about side effects) compared to January 2013, occurred on both units: $43 \%(\mathrm{p}=0.007)$ and $13.3 \%(\mathrm{p}=0.013)$ increase in the intervention and control units, respectively (Figure 4 ).

Scores from 2013 were compared to the average scores in 2012 from both units. Increased patient counseling resulted in a non-significant change in the scores for both questions when comparing average scores from 2012 versus 2013. A total of 144 surveys were completed in 2012 and 25 surveys completed in 2013 on the intervention unit. The percentage of patients answering "always" to question 1 was $66 \%$ vs. $72 \%$ in 2013 ( $\mathrm{p}=0.374)$. The percentage of patients answering "always" to question 2 was $29.2 \%$ in 2012 vs. $25 \%$ in 2013 ( $\mathrm{p}=0.889$ ). A total of 177 surveys were completed for the control unit in 2012 and 30 surveys were completed in 2013. The total percentage of patients answering "always" to question 1 was $79.2 \%$ in 2012 vs. $86.7 \%$ in 2013 ( $\mathrm{p}=0.87$ ). The total percentage of patients answering "always" to question 2 was $39.8 \%$ in 2012 vs. $53.3 \%$ in 2013 ( $\mathrm{p}=0.9$ ). No significant differences in patient satisfaction with respect to either question one $(p=0.326)$ or two ( $p=0.527)$ were noted between the units from 2013 (25 surveys from the intervention unit and 30 from the control unit) (Figure 4).

\section{Medication Reconciliation Intervention}

MR was successfully completed for each patient admitted on the intervention unit and documented in the patients' chart. The first phase of the MR survey was administered to staff working on the intervention unit approximately 6 months prior to the study month. A total of 28 individuals ( $71 \%$ nurses) completed the first phase of the MR survey. The second phase of the MR survey was completed within approximately 2 weeks of the completion of the MR intervention. Only 13 (46\%) of the individuals completed the second phase of the survey. We were unable to contact certain nurses who had left the health-system, or were relocated to a different floor within the hospital, and therefore they would not be able to adequately assess MR on the intervention unit. Four of the ten questions that assessed various aspects of the MR process trended towards a significant change of improvement when compared before and after the study. These included decreased number of medication misadventures $(p=0.107)$, increased efficiency of patient admission ( $p$ $=0.157)$, decreased interference with patient discharge $(\mathrm{p}=0.157)$, and decreased total time to complete the discharge process $(\mathrm{p}=0.058)$.

\section{FTE Analysis}

A total of 256 counseling sessions were completed during the 27 day study. The pharmacist spent an average of 10 minutes per counseling session on the intervention unit and an average of 13 minutes per session on the control unit. Each patient on the intervention unit received an average of 2.7 counseling sessions versus one counseling session on the control unit. A total of 16 extra minutes were required to counsel patients daily throughout their three day admission if they were on the intervention unit versus the control unit.

A total of 1613 orders were verified on the intervention unit by the PI during the hours of $10 \mathrm{am}$ and $6 \mathrm{pm}$. Seventy-four percent (1194) orders were verified successfully by the PI. Approximately 15 orders were not verified by the PI daily. The investigators discovered this was likely caused by pharmacists who were working "as needed" within our institution and were unaware of the research project. Education was provided to those individuals throughout the month to reinforce the need to defer the orders to the primary investigator.

An FTE cost-analysis was completed to determine the potential financial requirement to incorporate the type of pharmacy practice model conducted in this study throughout our 500-bed hospital. We utilized an additional 16 minutes of counseling would be required per each patient's three day admission on the intervention unit versus the control unit. Based on the assumptions stated in our methods, we calculated our required FTEs based on these assumptions as well as the total number of pharmacists we would need to be placed in each adult medicine unit throughout the hospital. Therefore, we would require an additional 4 to7 FTEs (approximately $\$ 400,000-\$ 700,000$ ) to implement this practice model throughout our institution. We could utilize a minimum of 4 additional FTEs if we allowed cross-coverage between units where our volume was lower.

\section{Discussion}

We found that counseling by a pharmacist statistically significantly improved side effect communication when provided to a patient multiple times versus once throughout their admission. Additionally, a trend was seen towards improvement in overall medication communication scores. Communication about the purpose of medications decreased after the study intervention on both units. This may be explained by the fact that the actual questions on the HCAHPS survey identify "hospital staff" in general as being responsible for the medication communication. Patients likely considered the communication by nursing and physician staff instead of pharmacists alone. Patients consider this question to pertain to the direct administration of medications, and how often a nurse explains to them the use of a medication when it is administered. Therefore, pharmacists can make an impact on these endpoints, but ultimately, multiple healthcare providers contribute to the medication communication HCAHPS survey results. Our institution can rework our patient counseling processes and continue to work with our nursing team to improve medication communication and remind each other as colleagues to touch on these points when administering or discussing medications.

The impact of increased counseling by pharmacists was assessed in a study including 125 patients with low literacy [8]. Patients were 
Citation: Huebner M, Temple-Cooper ME, Lagzdins M, Yeh J (2013) A Pilot Study Evaluating the Effect of Daily Education by a Pharmacist on Medication Related HCAHPS Scores and Medication Reconciliation Satisfaction. J Biosafety Health Educ 1: 105. doi:10.4172/2332-0893.10 00105

Page 5 of 6

randomized 1:1 to have medication counseling completed by either a pharmacist or a nurse or physician taking care of the patient. The patients randomized to the pharmacist group received counseling upon admission, discharge, and follow-up after discharge. A survey was provided to patients to assess the utility of the different components of the intervention after the intervention was completed. Seventytwo percent of the patients reported it was "very helpful" to talk to the pharmacist about their medications. Sixty-three percent and $72 \%$ of patients said the intervention was "very helpful" in preventing and managing side effects and understanding how to take their medications, respectively [8]. Our study had similar results, although the number of patients that completed the HCAHPS survey was much lower. The survey provided to patients in the aforementioned survey identified the pharmacist as the healthcare provider in the question [8].

A MR intervention was included in this study to improve our current processes within the hospital. The key difference with our study was that a pharmacist completed the process as opposed to nursing staff. Authors conducted a study to evaluate the effect of pharmacist driven medication reconciliation on preventable medication errors post-discharge in low-literacy patients.9 Patients were randomized 1:1 to have medications reconciled by a pharmacist or a nurse or physician taking care of them. Authors included 851 patients, in which 432 (51\%) experienced one or more clinically important medication errors during 30 days after hospital discharge. Mean number of medication errors were similar per patient in the intervention and control groups, 0.87 per patient versus 0.95 , respectively $(p=0.92)$. Authors concluded that no statistical difference existed between the prevention of medication errors when a pharmacist completed medication reconciliation versus a physician or nurse [9]. Our survey results showed a trend towards reduction in medication errors post intervention by a pharmacist. This data is based on opinion, and actual incidence of medication errors is important to study in the future similar to the aforementioned study [9].

Our FTE analysis was completed to accurately reflect the financial burden that may be required to implement a pharmacy practice model within our hospital similar to the one utilized in our study: a pharmacist providing daily counseling, completing $\mathrm{MR}$, and verifying all medication orders for a patients on a 25 bed unit. The major difference between the practice model utilized in our study and the one that would be implemented within our hospital, is that patients being discharged to skilled nursing facilities and long-term care facilities would not be excluded from MR and patient counseling. This likely will increase the amount of time spent completing MR and patient counseling by the pharmacist and may limit the time a pharmacist could spend with each patient, thus affecting the quality of the counseling sessions.

Several limitations existed within our study including differences between the patient populations on the intervention and control units, low return on completed HCAHP and medication reconciliation satisfaction surveys, barriers to complete medication reconciliation interventions, and limited ability to apply our FTE cost analysis elsewhere within our institution. We attempted to include the most similar units within the hospital when choosing our intervention and control units, although there were inherit differences that could have affected our results. The primary differences included admitting indications. On the control unit, most patients were admitted postorthopedic surgery. Medication communication often included pain management topics as well as bowel regimen control. These patients require a considerably larger amount of pain medications compared to the control unit, where the primary indication was gastrointestinal related surgeries. Patients were discharged post-operatively sooner on the control unit (within 24-48 hours) than the intervention unit (within 72 hour average or longer), indicating the need for a longer duration of pain management post-operatively on the intervention unit. Increased pain levels as well as pain medication use, could have affected the patients' overall experience within the hospital, and in turn contributed to their survey results. Additionally, we did not meet our required number of completed patient surveys to accurately measure the impact of daily counseling on this patient population.

Although, MR was reviewed upon admission for each patient included on the intervention unit, a number of barriers may have limited the success of this process. The medication orders for most MR interventions were already approved by the physician by the time the patient arrived on the intervention unit. This required the PI to make MR changes only after speaking to the physician. If the patient's MR was completed overnight, the PI had to make the changes the next day, and the patient may have received medications in error in the mean time. The greatest disadvantages to MR conducted in this manner, included increased time requirement to contact the physician and inability to catch the errors before administration to the patient (i.e. if patient was admitted overnight). Ideally, the MR could be completed either in the emergency room by a pharmacist or by a pharmacist on the floor prior to physician orders being placed. We hope to conduct MR in this manner in the future. Additionally, the second phase of our MR survey was not completed by over half of the staff members who completed phase I of the survey. This limited our ability to fully evaluate the effect of MR completed by a pharmacist versus the nursing staff.

Lastly, our cost analysis was based on the average length of stay on one of our adult medical-surgical units which was 3 days. This does not reflect the varying length of stays throughout our other adult medicine units; therefore the additional time requirement per patient admission for daily counseling may be greater in other hospital units.

\section{Conclusion}

Our data indicated that daily counseling by a pharmacist can improve medication communication related HCAHPS scores, and thus improve patient care. Although our results were limited by low numbers of completed HCAHPS and MR satisfaction surveys, our trends towards significance indicates the positive impact this practice model could have on patient care if implemented into practice. Our study provides data for a larger study to completely validate the effectiveness of pharmacists in improving medication communication and the medication reconciliation process. In the future, when medication reconciliation is completed, pharmacists should document the number of changes made to the medication regimen and the incidence of medication errors should be evaluated. Our FTE analysis indicated that additional time will be required to counsel daily throughout admission on the intervention unit. The counseling requirements and lengths of stay vary from unit to unit within our hospital and therefore this practice model should be tested on other adult medicine units within our facility. Further study is needed to assess the impact of a pharmacist on patient readmission rates and improved medication communication when a program similar to ours is instituted.

\section{References}

1. Griffith NL, Schommer JC, Wirsching RG (1998) Survey of inpatient counseling by hospital pharmacists. Am J Health Syst Pharm 55: 1127-1133.

2. Federal Register, Daily Journal of the United States Government. Medicare Program; Hospital Inpatient Value-Based Purchasing Program. A Rule by the Centers for Medicare \& Medicaid Services. 
Citation: Huebner M, Temple-Cooper ME, Lagzdins M, Yeh J (2013) A Pilot Study Evaluating the Effect of Daily Education by a Pharmacist on Medication Related HCAHPS Scores and Medication Reconciliation Satisfaction. J Biosafety Health Educ 1: 105. doi:10.4172/2332-0893.10 00105

Page 6 of 6

3. Centers for Medicare \& Medicaid Services, Baltimore, MD.

4. Kramer JS, Hopkins PJ, Rosendale JC, Garrelts JC, Hale LS, et al. (2007) Implementation of an electronic system for medication reconciliation. Am J Health Syst Pharm 64: 404-422.

5. Temple ME, Jakubecz MA, Link NA (2013) Implementation of a training program to improve pharmacy services for high-risk neonatal and maternal populations. Am J Health Syst Pharm 70: 144-149.

6. Physician's orders: accepting, writing, transcribing (2012) Euclid, Hillcrest, and South Pointe Hospitals Hospital Policy Manual Clinical Services.
7. Systat Software. Cranes Software International, Ltd.

8. Cawthon C, Walia S, Osborn CY, Niesner KJ, Schnipper JL, et al. (2012) Improving care transitions: the patient perspective. J Health Commun 17 Supp 3: 312-324.

9. Kripalani S, Roumie CL, Dalal AK, Cawthon C, Businger A, et al. (2012) Effect of a pharmacist intervention on clinically important medication errors after hospital discharge: a randomized trial. Ann Intern Med 157: 1-10. 